\title{
Correlation Between Fetal Weight Gain and Birth Weight with Blood Flow in the Uterine Arteries Calculated with the Pixelflux Technique
}

(๑)( $\odot \Theta$

Authors

Helene Caroline Arneberg1, *, Thea Anette Andersen1, *, Liv Lorås², Hans Torp³, Thomas Manfred Scholbach", Torbjørn Moe Eggebø1,2

\section{Affiliations}

1 Norwegian University of Science and Technology, Institute of Clinical and Molecular Medicine, Trondheim, Norway

2 Trondheim University Hospital (St. Olavs Hospital), National Center for Fetal Medicine, Trondheim, Norway

3 Norwegian University of Science and Technology, Department of Circulation and Medical Imaging, Trondheim, Norway

4 Leipzig Ultrasound Institute, Leipzig, Germany

Key words

fetus, obstetrics, pregnancy, uterus, ultrasound-color doppler

received 11.01 .2017

revised $\quad 07.11 .2017$

accepted 09.01.2018

Bibliography

DOI https://doi.org/10.1055/s-0044-102005

Ultrasound Int Open 2018; 4: E16-E22

(c) Georg Thieme Verlag KG Stuttgart · New York ISSN 2199-7152

Correspondence

Ms. Helene Caroline Arneberg, Medical Student Norwegian University of Science and Technology Institute of Clinical and Molecular Medicine

Olav Kyrres gate 11

Trondheim, 7030

Norway

Tel.: +47/412/07 057

heleneca@hotmail.com

\section{ABSTRACT}

Introduction The aim was to investigate correlations between fetal weight gain/day and birthweight with blood flow estimates in the uterine arteries calculated with the PixelFlux technique and with measurements from TAmax. We also aimed to examine the agreement between estimates using the two methods.

Material and methods We conducted a prospective observational pilot study in pregnancy week 24-25 in women with risk pregnancies referred to the fetal medical centre at St. Olavs Hospital, Trondheim, Norway from March 2016 to June 2016. Blood flow in the uterine arteries was calculated using timeaveraged peak velocity (TAmax) and the PixelFlux technique. PixelFlux is a method based on pixelwise calculation of spatially angle-corrected velocities and areas of all pixels inside a vessel during a heart cycle.

Results The mean flow calculated from PixelFlux and TAmax was $811 \mathrm{ml} /$ minute and $787 \mathrm{ml} /$ minute, respectively. The intra-class correlation coefficient was 0.83 ( $95 \% \mathrm{Cl} 0.72-0.90)$ and limits of agreement were $-441 \mathrm{ml} /$ minute $(95 \% \mathrm{Cl}-558$ to $-324 \mathrm{ml} /$ minute) to $489 \mathrm{ml} /$ minute $(95 \% \mathrm{Cl} 372$ to $606 \mathrm{ml} /$ minute). We observed a significant correlation between mean flow calculated from PixelFlux and birthweight $(r=0.41$; $p<0.01)$ and between flow calculated from PixelFlux and weight gain/day $(r=0.33 ; p=0.02)$. Calculation based on TAmax was significant correlated to birthweight $(r=0.34$; $p=0.02$ ), but not to weight-gain/day. Pulsatile index was not correlated to flow, birthweight or fetal weight-gain/day.

Conclusions We found significant correlations between estimated blood flow in the uterine arteries using the PixelFlux technique with fetal weight-gain/day and with birthweight. Estimates from two methods showed good agreement.

\footnotetext{
* The two first authors contributed equally to this work.
} 


\section{Abbreviations}

PI pulsatility index

$\mathrm{RI}$ resistance index

TAmax time-averaged peak velocity

BMI body mass index

R correlation coefficient

\section{Introduction}

The volume of the utero-placental blood flow is an important parameter in assessing normal placental function and fetal development [1]. Using ultrasound color Doppler, it is possible to assess the flow resistance in the placenta [2]. The pulsatility index ( $\mathrm{PI})$ and resistance index (RI) are commonly used to quantify the ratio of blood flow velocities in the uterine artery and the placenta [3]. High resistance based on high PI values and notches in the uterine arteries are used as predictors of adverse maternal and fetal outcomes $[1,4-7]$. It is assumed that a precise measurement of the total blood flow $(Q)$ would be the best way to assess placental function and well-being of the fetus. Blood flow can be calculated from the diameter of the vessel and time-averaged maximum velocity (TAmax) or the mean velocity (TAmean) using the formula $0.5 *$ TAmax $(\mathrm{cm} / \mathrm{s}){ }^{*}$ cross section area of the vessel $(\mathrm{CSA})\left(\mathrm{cm}^{2}\right)$ * 60. CSA is calculated as $\pi{ }^{*}$ (diameter $\left./ 2\right)^{2}$ assuming that the vessels are circular [8-15]. It has recently been suggested that color Doppler with the PixelFlux technique can be used to calculate flow by examining the area and color of each pixel in a vessel section $[16,17]$. It is a post-processing method in which each pixel is assigned a spatial angle correction. The spatial angle is calculated by a mathematical algorithm of the PixelFlux technique. This algorithm uses the shape of the elliptical vessel and its orientation in the frontal plane to extract the angle of the vessel with the frontal as well with the sagittal plane. After calculation of the spatial angle,

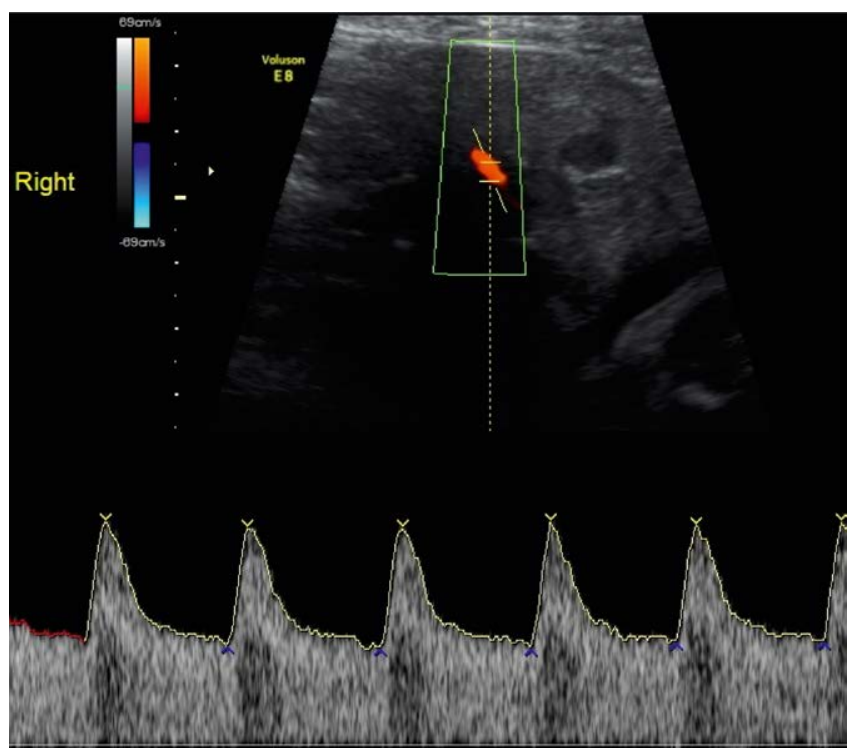

- Fig. 1 Flow calculated from TAmax (time-averaged maximum velocity) using the formula $0.5^{*} \operatorname{TAmax}(\mathrm{cm} / \mathrm{s}){ }^{*}$ cross section area of the vessel $(\mathrm{CSA})(\mathrm{cm} 2) * 60$. CSA was calculated as $\pi{ }^{*}$ (diameter $/ 2)^{2}$. each color pixel is corrected and the flow volume and the area corresponding to each pixel are calculated. All pixels during a heart cycle are added, and the total flow is estimated. The PixelFlux software is a commercial software application based on the recording made by conventional Doppler and analysed off-line from a twodimensional video-clip [18].

The aims of the study were to investigate correlations between fetal weight gain and birth weight with blood flow estimates in the uterine arteries calculated with the PixelFlux technique and with measurements from TAmax. We also aimed to examine agreement between estimates using the two methods.

\section{Materials and Methods}

We conducted a prospective observational pilot study of women with single pregnancies who, due to high-risk pregnancies, were referred to the fetal medical center at St. Olavs Hospital, Trondheim, Norway, from March 2016 to June 2016. They were referred to have an examination of the pulsatility index (PI) with Doppler ultrasound in the uterine arteries in gestational week 24-25. An extended examination including fetal weight estimation and measurements of blood flow in the uterine arteries was performed. The outcome measures were birth weight, weight gain/day from the ultrasound examination to delivery and preterm deliveries. All women gave written consent and the local ethics committee approved the study (Rek Midt 2015/2304).

Transabdominal ultrasound measurements were performed using Voluson E8 ultrasound equipment (GE Medical Systems, Zipf, Austria) with a 3.5-7.5-MHz three-dimensional curved multifrequency transabdominal transducer. All measurements were carried out by specially trained midwives. Blood flow volume (ml/ minute) in the uterine arteries was first calculated from TAmax ( $\triangleright$ Fig. 1). The angle of the ultrasound waves to the vessels was corrected, and kept as close as possible to zero. The diameter of the vessel was measured in the systole on the color Doppler image using the method published in other studies $[11,15,19]$. The blood flow in the two uterine arteries was summarized.

Thereafter, the blood flow was calculated using the PixelFlux method ( $>$ Fig. 2). The flow volumes of all pixels inside a vessel's section were added to calculate the flow volume of the vessel during a heart cycle. $>$ Fig. 3 and $>$ Video. 1 illustrate variation during a heart cycle. All the calculations and measurements were done automatically by the PixelFlux program [18].

Fetal weight in the second trimester was estimated using the mean of three measurements of the biparietal diameter (BPD) and abdominal circumference (AC). We calculated fetal weight using the algorithm implemented in eSnurra [20], as recommended by the Norwegian Directory of Health. The birth weight was obtained immediately after birth. The mean fetal weight gain/day was calculated as the difference between the birth weight and estimated fetal weight in the second trimester, and thereafter compared to the remaining days in pregnancy from the ultrasound examination to delivery.

\section{Statistical analyses}

Categorical variables were compared using Chi-square test and continuous variables using Mann-Whitney U-test and linear regres- 
sion. In the regression analyses we adjusted for possible confounders such as parity, maternal age, body mass index (BMI) and systolic blood pressure. Correlation between methods was analyzed using the intra-class correlation coefficient. If zero was inside the $95 \% \mathrm{Cl}$ of the mean difference between methods, no bias was assumed. The analysis of inter-method agreement was performed using limits of agreement as described by Bland and Altman [21]. $\mathrm{P}<0.05$ was considered statistically significant. Statistical analyses were performed with SPSS Statistics for Mac, v. 23.0 Armonk, NY: IBM Corp.

\section{Results}

In all, 60 women were included in the study; 7 of these were excluded due to a suboptimal insonation angle, 3 because fetal weight estimation was not performed and 3 because we were missing information about birth weight, leaving 47 women in the study population. The characteristics of the study population are presented in > Table $\mathbf{1}$.

The mean flow calculated from PixelFlux was $811 \mathrm{ml} /$ minute (median 777, range 209-1988 $\mathrm{ml} /$ minute) and the mean flow using calculation from TAmax was $787 \mathrm{ml} /$ minute (median 710, range $179-2120 \mathrm{ml} /$ minute). The mean difference was $24 \mathrm{ml} /$ minute ( $95 \% \mathrm{Cl}-45$ to $94 \mathrm{ml} /$ minute) and no significant difference between the two methods was observed because the $\mathrm{Cl}$ intervals of the mean difference were crossing zero. The agreement between the mean flows from the two methods is presented as a scatter plot in - Fig. 4 and as a Bland Altman plot in $\mathbf{F i g}$. 5. The intra-class correlation coefficient was $0.83(95 \% \mathrm{Cl} 0.72-0.90)$ and the limits of agreement were $-441 \mathrm{ml} /$ minute $(95 \% \mathrm{Cl}-558$ to $-324 \mathrm{ml} /$ minute) to $489 \mathrm{ml} /$ minute $(95 \% \mathrm{Cl} 372$ to $606 \mathrm{ml} /$ minute). Details are presented in $>$ Fig. 6.

We observed a significant correlation between birth weight and the mean flow calculated with PixelFlux $(r=0.41 ; p<0.01)$ as presented in > Fig. 7, and between weight gain/day and the flow calculated with PixelFlux $(r=0.33 ; p=0.02)$ as presented in - Fig. 8. The correlations remained significant after adjusting for maternal age, BMI, parity and systolic blood pressure $(p=0.01$ and $p=0.02$, respectively). The correlations between flow calculated from TAmax and birth weight or weight gain/day are presented in - Table 2. We did not observe any significant correlation between PI and flow calculated with PixelFlux $(r=-0.03 ; p=0.83)$ as illustrated in > Fig. 9, or between PI and RI with birth weight or weight gain/ day ( $\triangleright$ Table 2). For every $100-\mathrm{mL} / \mathrm{min}$ increase in total blood flow calculated from PixelFlux and from TAmax, there was an increase in birth weight of 70 grams and $62 \mathrm{~g}$, respectively.

Two women delivered preterm; one in week 26 and one in week 27 , both due to spontaneous contractions. These two women did not have preeclampsia or hypertension, and the fetal weight was appropriate for gestational age. In these women, the flow calculated with PixelFlux was 307 and $209 \mathrm{ml} /$ minute, respectively, the flow calculated from TAmax was 388 and $201 \mathrm{ml} / \mathrm{min}$, respectively, and the mean $\mathrm{PI}$ was 0.87 and 0.88 , respectively.

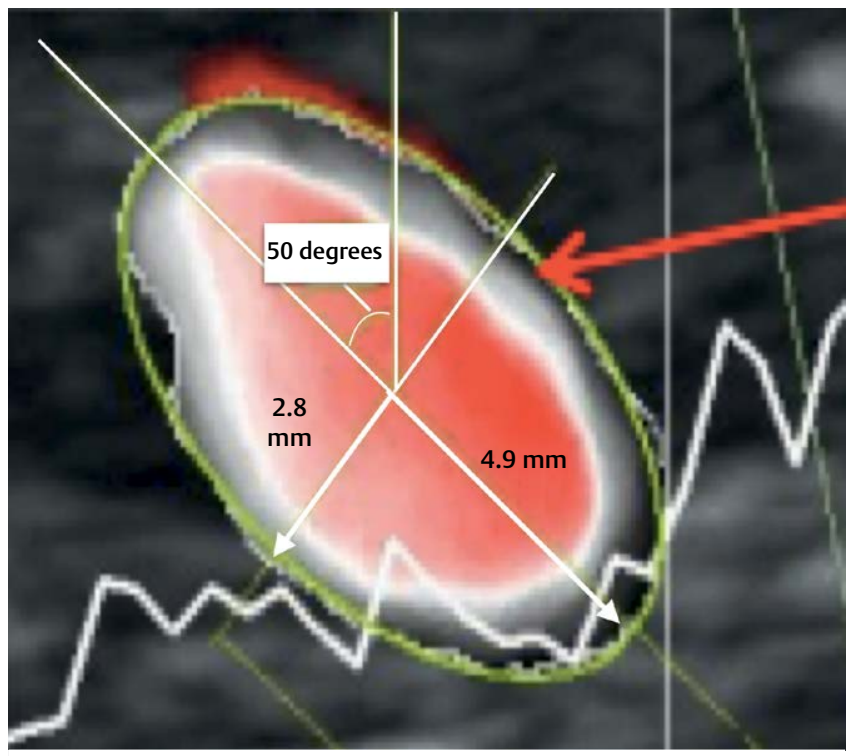

-Fig. 2 PixelFlux is a Doppler ultrasound method. Every pixel represents a spatial angle corrected velocity, which is coded by a color. All velocities as well as the area of all pixels are summarized and the total flow is calculated during a full heart cycle.
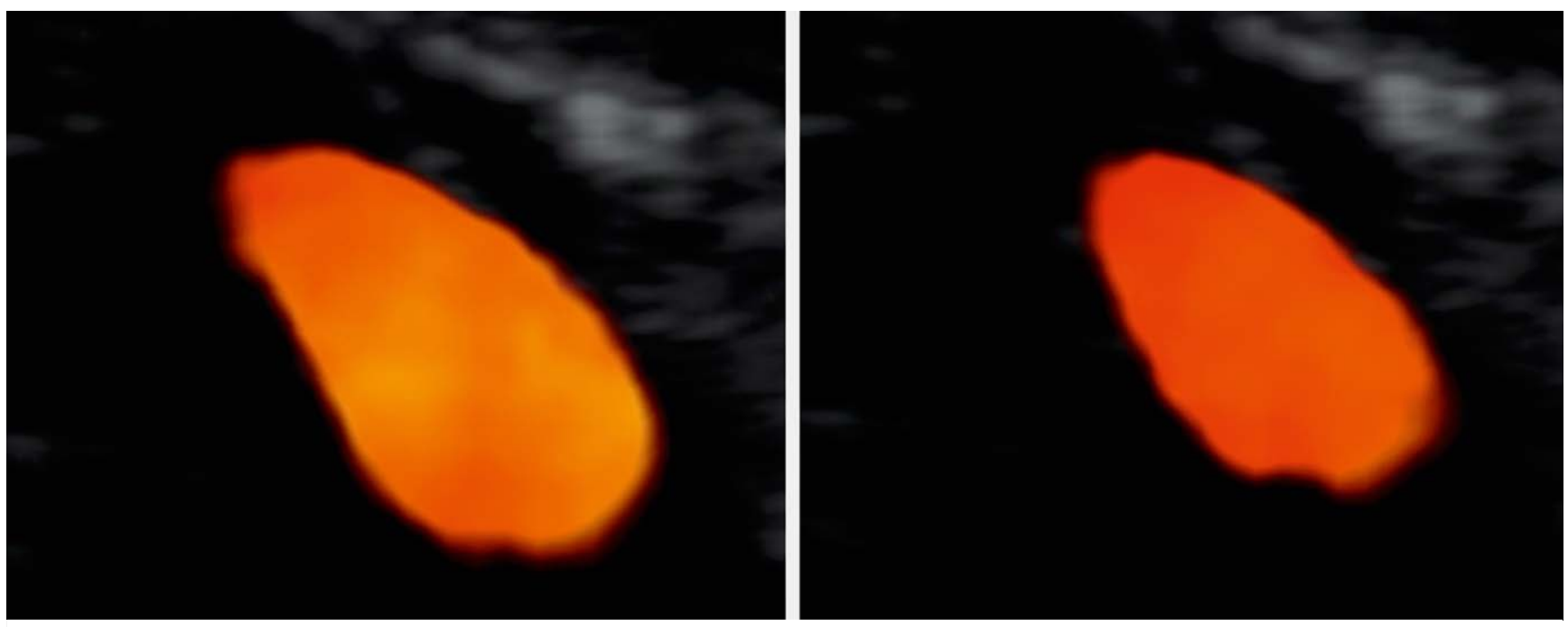

-Fig. 3 Variation in velocity (color) in left uterine artery during a heart cycle (systole to left and diastole to right). 


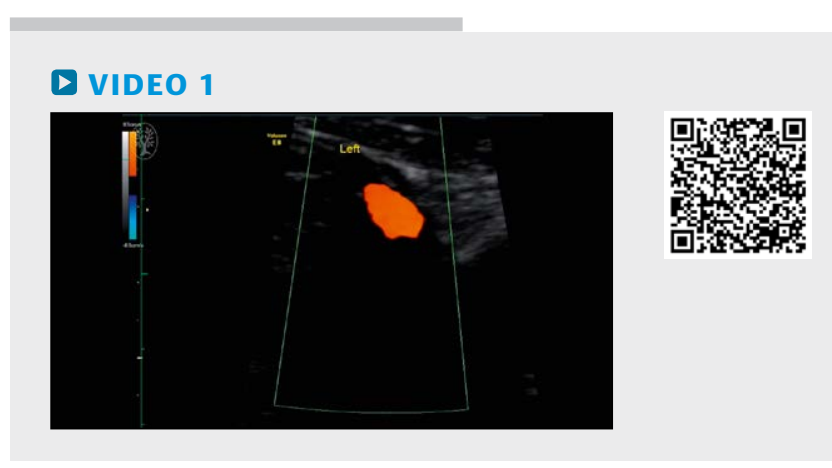

- Video. 1 Variation in velocity (color) in left uterine artery during a heart cycle. The color bar is shown to the left.

- Table 1 Characteristics of study population.

\begin{tabular}{|l|c|c|}
\hline & Median & Range \\
\hline Maternal characteristics & & \\
\hline Maternal age (years) & 32 & $21-45$ \\
\hline BMI & 26 & $20-48$ \\
\hline Systolic blood pressure $(\mathrm{mmHg})$ & 114 & $90-141$ \\
\hline Diastolic blood pressure $(\mathrm{mmHg})$ & 70 & $46-91$ \\
\hline Pregnancy characteristics & & \\
\hline Pregnancy duration (days) & 280 & $186-293$ \\
\hline Characteristics of the newborn & & \\
\hline Weight gain per day $(\mathrm{g})$ & 26 & $13-37$ \\
\hline Birth weight $(\mathrm{g})$ & 3445 & $875-5000$ \\
\hline Apgar score 1 min & 9 & $3-10$ \\
\hline Apgar score 5 min & 10 & $4-10$ \\
\hline Apgar score 10 min & 10 & $8-10$ \\
\hline pH in umbilical artery & 7.24 & $7.06-7.38$ \\
\hline
\end{tabular}

\section{Discussion}

The main finding in the study was a significant correlation between estimated blood flow in the uterine arteries using the PixelFlux technique with fetal weight gain/day and with birth weight. TAmax calculations achieved a significant correlation with birth weight. We observed a good correlation between the PixelFlux and the TAmax technique to estimate blood flow in the uterine arteries in pregnancy week 24-25.

The pulsatility index is the preferred variable in clinical practice because it is easy to measure, and independent of the angle of insonation [22]. A high PI in the uterine arteries during pregnancy reflects high resistance in the placenta, and a high Pl in the second trimester has been associated with increased risk of fetal growth restriction [2], preeclampsia [1,3], placental infarction [7] and adverse fetal outcome [6]. In our study, however, we did not observe any association between $\mathrm{Pl}$ and fetal growth. Pl and blood flow were not associated, and placental flow might thus be more important for fetal growth than placental resistance.

Calculation of volume blood flow is desirable, but noninvasive techniques are challenging, especially in small vessels and in pul-

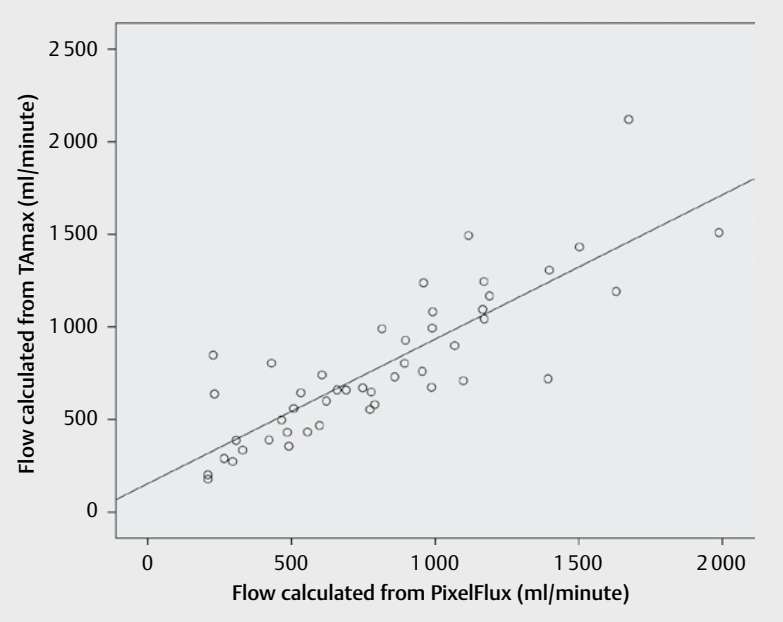

- Fig. 4 Association between flow calculated from TAmax and PixelFlux (Regression equation: $y=155+0.78 x$ ).

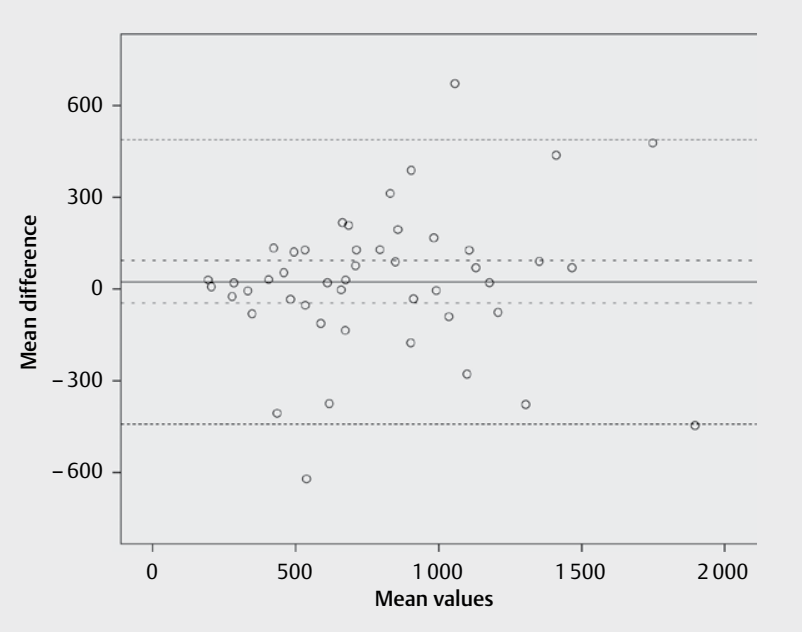

-Fig. 5 Bland-Altman plot for inter-method agreement of total uterine artery flow based on time-averaged peak velocity and the PixelFlux technique. Mean difference in $\mathrm{ml} / \mathrm{min}$ (continues line) with $95 \%$ confidence interval (separated dots) and $95 \%$ limits of agreement (tight dots) (i.e., mean difference $\pm 1.96 \mathrm{SD}$ ) are shown.

sating arteries [19]. Using standard calculations from TAmax, it is assumed that the mean cross-sectional velocity is $0.5 \times$ TAmax. The constant 0.5 is used to calculate flow in tubes with a parabolic velocity profile [8].

The PixelFlux method calculates flow through at least one entire heart cycle, and an automatic spatial angle correction in both the sagittal and frontal plane is implemented in the computer program [18], assuming circular vessel geometry. The main advantage is that simple 2-dimensional imaging is sufficient to calculate 3-dimensional flow volumes (so-called "PixelFlux 243" technique). An oblique vessel section is made and a short video clip of the vessel is recorded. Asymmetric flow distribution as well as the changing vessel area and flow velocities during the heart cycle are detected automatically by the PixelFlux technique. However, turbulence is 


\begin{tabular}{|c|c|c|c|c|c|c|c|c|c|}
\hline \multirow{3}{*}{ Inter method agreement } & \multirow{3}{*}{$\begin{array}{c}\text { Mean } \\
799\end{array}$} & \multirow{3}{*}{$\begin{array}{c}\text { Median } \\
709\end{array}$} & \multirow{3}{*}{$\begin{array}{c}\text { Range } \\
\text { (194 to 1 897) }\end{array}$} & \multirow{3}{*}{$\begin{array}{c}\text { ICC }(95 \% C l) \\
0.83(0.72-0.90)\end{array}$} & \multicolumn{5}{|c|}{ Difference between the two observers } \\
\hline & & & & & Mean $(95 \% C l)$ & $1.96 S D$ & Lower limit (95\% Cl) & Upper limit (95\% CI) & Range \\
\hline & & & & & $24(-45$ to 94$)$ & 465 & $-441(-558$ to -324$)$ & 489 (372 to 606$)$ & (-621 to 672$)$ \\
\hline
\end{tabular}

Mean, median and range are calculated from the mean results from the two methods; ICC, intraclass correlation coefficient; SD, standard deviation.

- Fig. 6 Inter-method agreement of mean flow in uterine arteries calculated from TAmax and from PixelFlux.

still a problem and it is important to adjust pulse repetition frequency to avoid aliasing.

The total blood flow volumes in the uterine arteries increase during pregnancy [23]. Previous studies have shown varying blood flow volumes in the second trimester $[11,24,25]$. One reason for the variation in blood flow volumes may be that the vessel diameter has a great influence on calculated flow based on TAmax or TAmean, and it is difficult to measure the diameter of the uterine arteries using two-dimensional transabdominal sonography. It is easier to measure the vessel diameter on color Doppler images. However, the diameter might be slightly overestimated on Doppler images $[11,19]$. The variation might also be related to the insonation angle when the vessel diameter is measured.

Acharya et al. compared Doppler measurements of blood flow with actual flow using an invasive method in sheep and found good correlation [19]. They measured the vessel diameter on power Doppler images during systole. Konje et al. calculated the total uterine artery flow to be around $500 \mathrm{ml} / \mathrm{min}$ in pregnancy week 20 with increasing flow of $39 \mathrm{~mL} / \mathrm{min}$ per week from week 20 to 24 [11]. McKelvey et al. found the mean total blood flow in the uterine arteries to be more than $800 \mathrm{ml} / \mathrm{min}$ in pregnancy week 23-26 [15]. These findings correlate well with the findings in our study where we found total uterine artery blood flow to be around $800 \mathrm{ml} / \mathrm{min}$ and the mean gestation length at the time of the ultrasound examinations was 24 weeks and 4 days. In color Doppler acquisitions, the insonation angle should be as close as possible to zero degrees and we measured the vessel diameter on these images. This insonation angle is not optimal for measuring vessel diameter because the lateral resolution in ultrasound images is lower than the axial resolution. Thus, we might have overestimated the vessel diameter. However, our aim was not to calculate absolute flow, but to compare methods and to investigate correlations between flow and fetal growth.

The strengths of the study were that all women were examined in pregnancy week 24-25 and that one examiner used the PixelFlux technique and another examiner calculated flow using TAmax. The examiner using PixelFlux was blinded and not informed about clinical indications or about labor outcomes. The limitations of the study are its small study population and that it was a pilot study without a power calculation. It was sometimes challenging to achieve an optimal insonation angle of the uterine arteries and we had to exclude seven cases due to insufficient insonation angles. We do not have any gold standard for uterine artery blood flow measurement in humans. Invasive measurements during pregnancy would be unethical, and animal studies are necessary for validation of Doppler estimates.

In conclusion, we found significant correlations between estimated blood flow in the uterine arteries using the PixelFlux

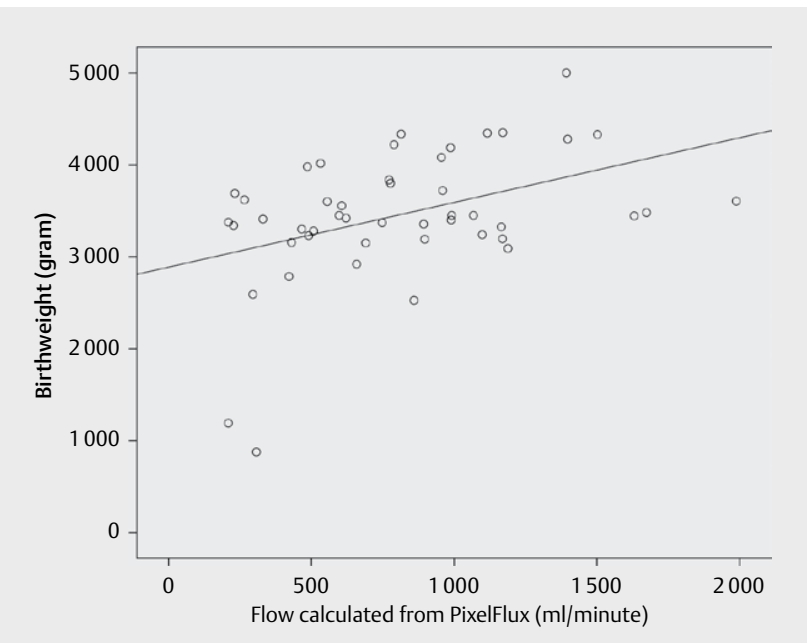

-Fig. 7 Association between flow calculated from PixelFlux and birthweight. (Regression equation: $y=2890+0.70 x$ ).

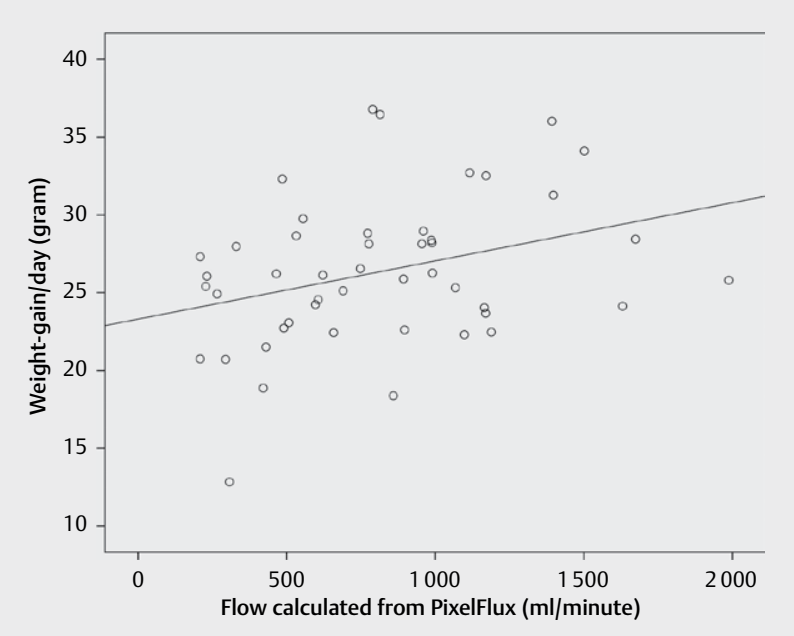

-Fig. 8 Association between flow calculated from PixelFlux and weight-gain/day (Regression equation: $y=23+0.004 x$ ).

technique with fetal weight gain/day and with birth weight. Estimates from the PixelFlux method and from TAmax showed good agreement. The PixelFlux method might be a promising tool for predicting pregnancy outcome. However, new and larger studies are necessary. 
- Table 2 Correlations between fetal growth and mean of ultrasound parameters from the two uterine arteries.

\begin{tabular}{|c|c|c|c|c|c|c|c|c|}
\hline \multirow{2}{*}{ Correlation to } & \multicolumn{2}{|c|}{ PixelFlux } & \multicolumn{2}{|c|}{ TAmax } & \multicolumn{2}{|c|}{ PI } & \multicolumn{2}{|c|}{ RI } \\
\hline & $\mathbf{r}$ & p-value & $\mathbf{r}$ & p-value & $\mathbf{r}$ & p-value & $\mathbf{r}$ & p-value \\
\hline Birth weight & 0.42 & $<0.01$ & 0.34 & 0.02 & -0.25 & 0.09 & -0.23 & 0.13 \\
\hline Weight gain/day & 0.33 & 0.02 & 0.29 & 0.05 & -0.18 & 0.22 & -0.15 & 0.33 \\
\hline
\end{tabular}

TAmax: time-averaged peak velocity; Pl: pulsatile index; r: correlation coefficient.

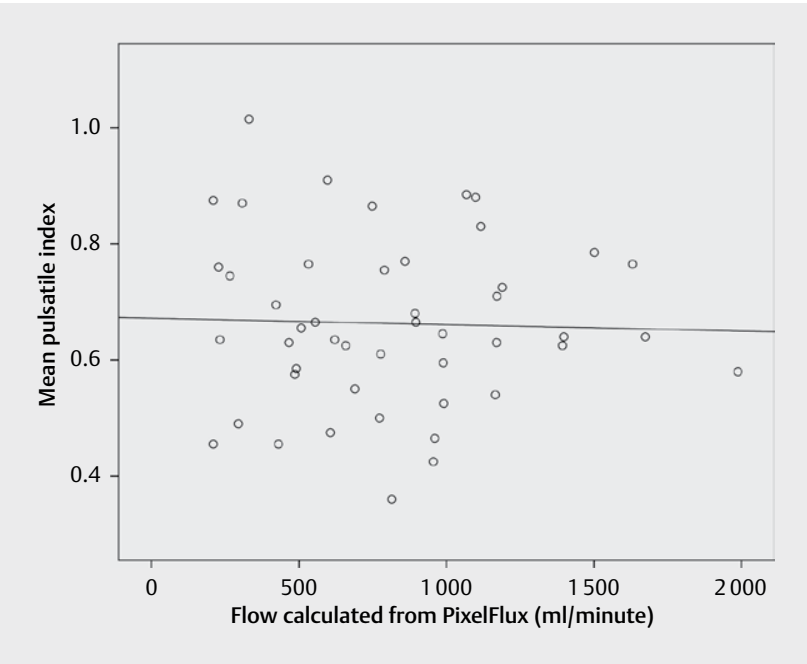

- Fig. 9 Association between flow calculated from PixelFlux and pulsatile index (Regression equation: $y=0.67-0.00001 x$ ).

\section{Acknowledgements}

We would like to thank Bente Simensen, Ingunn Aas and Eva Leknes Jensen for performing the ultrasound examinations and Nancy Eik-Nes for important contribution in revising the manuscript.

\section{Conflict of Interest}

Thomas Scholbach is the scientific advisor of the Chameleon-Software $\mathrm{GmbH}$.

\section{References}

[1] Khalil A, Garcia-Mandujano R, Maiz N, Elkhouli M, Nicolaides KH. Longitudinal changes in uterine artery Doppler and blood pressure and risk of pre-eclampsia. Ultrasound Obstet Gynecol. 2014; 43: 541-547

[2] Olofsson P, Laurini RN, Marsal K. A high uterine artery pulsatility index reflects a defective development of placental bed spiral arteries in pregnancies complicated by hypertension and fetal growth retardation. Eur J Obstet Gynecol Reprod Biol 1993; 49: 161-168

[3] van Asselt K, Gudmundsson S, Lindqvist P, Marsal K. Uterine and umbilical artery velocimetry in pre-eclampsia. Acta Obstet Gynecol Scand. 1998; 77: 614-619
[4] Thuring A, Laurini R, Marsal K. Uterine venous blood flow in normal and complicated pregnancies: A methodological study. Ultrasound Obstet Gynecol. 2010; 35: 462-467

[5] Hernandez-Andrade E, Brodszki ], Lingman G, Gudmundsson S, Molin J, Marsal K. Uterine artery score and perinatal outcome. Ultrasound Obstet Gynecol. 2002; 19: 438-442

[6] Poon LC, Volpe N, Muto B, Yu CK, Syngelaki A, Nicolaides KH. Second-trimester uterine artery Doppler in the prediction of stillbirths. Fetal Diagn Ther. 2013; 33: 28-35

[7] Orabona R, Donzelli CM, Falchetti M, Santoro A, Valcamonico A, Frusca T. Placental histological patterns and uterine artery Doppler velocimetry in pregnancies complicated by early or late pre-eclampsia. Ultrasound Obstet Gynecol. 2016; 47: 580-585

[8] Flo K, Wilsgaard T, Acharya G. Agreement between umbilical vein volume blood flow measurements obtained at the intra-abdominal portion and free loop of the umbilical cord. Ultrasound Obstet Gynecol. 2009; 34: 171-176

[9] Flo K, Wilsgaard T, Acharya G. Longitudinal reference ranges for umbilical vein blood flow at a free loop of the umbilical cord. Ultrasound Obstet Gynecol. 2010; 36: 567-572

[10] Flo K, Wilsgaard T, Acharya G. A new non-invasive method for measuring uterine vascular resistance and its relationship to uterine artery Doppler indices: A longitudinal study. Ultrasound Obstet Gynecol. 2011; 37: 538-542

[11] Konje JC, Kaufmann P, Bell SC, Taylor DJ. A longitudinal study of quantitative uterine blood flow with the use of color power angiography in appropriate for gestational age pregnancies. Am J Obstet Gynecol. 2001; 185: 608-613

[12] Konje JC, Howarth ES, Kaufmann P, Taylor DJ. Longitudinal quantification of uterine artery blood volume flow changes during gestation in pregnancies complicated by intrauterine growth restriction. BJOC 2003; 110: 301-305

[13] Bower S, Vyas S, Campbell S, Nicolaides KH. Color Doppler imaging of the uterine artery in pregnancy: Normal ranges of impedance to blood flow, mean velocity and volume of flow. Ultrasound Obstet Gynecol. 1992; 2: 261-265

[14] Dickey RP, Hower JF. Ultrasonographic features of uterine blood flow during the first 16 weeks of pregnancy. Hum Reprod. 1995; 10: $2448-2452$

[15] McKelvey A, Pateman K, Balchin I, Peebles DM, Rodeck CH, David AL. Total uterine artery blood volume flow rate in nulliparous women is associated with birth weight and gestational age at delivery. Ultrasound Obstet Gynecol. 2017; 49: 54-60

[16] Scholbach T, Heien C, Eggebo TM. Umbilical vein vasomotion detected in vivo by serial three-dimensional pixelwise spatially angle corrected volume flow measurements. Ultrasound Obstet Gynecol. 2015 
[17] Scholbach T, Fersis N, Stolle J. Significant differences of fetal blood supply in fetuses of different weight classes demonstrated by the novel method of three-dimensional pixelwise fetal volume flow measurements (PixelFlux-method). Ultrasound Obstet Gynecol. 2012; 40: 118

[18] Scholbach J, Scholbach T.PixelFlux. Chameleon Software, Available online at: http://www.chameleon-software.de/en/home.php (accessed Novemeber 7, 2017)

[19] Acharya G, Sitras V, Erkinaro T, Makikallio K, Kavasmaa T, Pakkila M et al. Experimental validation of uterine artery volume blood flow measurement by Doppler ultrasonography in pregnant sheep. Ultrasound Obstet Gynecol. 2007; 29: 401-406

[20] Gjessing HK, Grottum P, Okland I, Eik-Nes SH. Fetal size monitoring and birth-weight prediction: A new population-based approach. Ultrasound Obstet Gynecol. 2017; 49: 500-507

[21] Bland JM, Altman DG. Applying the right statistics: analyses of measurement studies. Ultrasound Obstet Gynecol. 2003; 22: 85-93
[22] Li N, Ghosh G, Gudmundsson S. Uterine artery Doppler in high-risk pregnancies at 23-24 gestational weeks is of value in predicting adverse outcome of pregnancy and selecting cases for more intense surveillance. Acta Obstet Gynecol Scand. 2014; 93: 1276-1281

[23] Thoresen M, Wesche J. Doppler measurements of changes in human mammary and uterine blood flow during pregnancy and lactation. Acta Obstet Gynecol Scand. 1988; 67: 741-745

[24] Ferrazzi E, Rigano S, Padoan A, Boito S, Pennati G, Galan HL. Uterine artery blood flow volume in pregnant women with an abnormal pulsatility index of the uterine arteries delivering normal or intrauterine growth restricted newborns. Placenta 2011; 32: 487-492

[25] Rigano S, Ferrazzi E, Boito S, Pennati G, Padoan A, Galan H. Blood flow volume of uterine arteries in human pregnancies determined using $3 \mathrm{D}$ and bi-dimensional imaging, angio-Doppler, and fluid-dynamic modeling. Placenta 2010; 31: 37-43 